\title{
Prevalência de Constipação Intestinal entre Estudantes de Medicina de uma Instituição no Noroeste Paulista
}

\author{
Prevalence of Constipation Among Medical Students of an Institution in \\ Northwestern São Paulo State
}

\author{
CAREN TRISÓGLIO ${ }^{1}$; CÍNTIA MARIA GARCIAMARCHI ${ }^{1}$; ULYSSES DOS SANTOS TORRES ${ }^{\mathbf{1}}$; JOÃO GOMES NETINHO² \\ 1. Graduandos em Medicina - Faculdade de Medicina de São José do Rio Preto (FAMERP); \\ 2. Chefe da Disciplina de Coloproctologia da FAMERP.
}

\begin{abstract}
TRISÓGLIO C; MARCHI CMG; TORRES US; NATINHO JG. Prevalência de constipação intestinal entre estudantes de medicina de uma instituição no noroeste paulista. Rev bras Coloproct, 2010;30(2): 203-209.

RESUMO: Introdução: A constipação intestinal (CI) é uma doença altamente prevalente em países ocidentais, apresenta etiologia complexa e multifatorial, e cursa com importantes impactos do ponto de vista médico e socioeconômico; é possível que profissionais da saúde e estudantes de medicina, dada a natureza de suas atividades profissionais e hábitos de vida, apresentem taxas de prevalência mais elevadas da doença, se comparados à população geral. Objetivos: Determinar a taxa de prevalência de CI entre estudantes de medicina de uma instituição de ensino segundo critérios diagnósticos estabelecidos na literatura e compará-la às taxas relatadas entre a população geral. Pacientes e Métodos: Realizou-se estudo transversal através da aplicação de questionários individuais a 360 estudantes de medicina de uma instituição do noroeste paulista, dos quais 150 (42\%) responderam; a definição de CI se deu através dos critérios de Roma III. Resultados: A taxa geral de prevalência de CI foi de 35\%; houve predominância da doença entre o sexo feminino $(55 \%$; $<<0,0001)$; estudantes do sexo feminino também apresentaram menor frequência de evacuações diárias e maior frequência de uso de laxativos $(\mathbf{p}<0,05)$. Conclusões: A prevalência de CI entre a população estudada foi superior à relatada entre a população geral em diversos estudos, e uma investigação mais complexa quanto às causas desse fato deverá ser objeto de novos estudos.
\end{abstract}

Descritores: Constipação intestinal; Trato Gastrointestinal; Epidemiologia; Prevalência; Estudantes de Medicina.

\section{INTRODUÇÃO}

A constipação intestinal $(\mathrm{CI})$ é uma doença polissintomática caracterizada por manifestações que podem interferir de maneira variada sobre as funções colônicas e anorretais. Com base em diferentes estudos epidemiológicos, estima-se que a doença acometa entre $15 \%$ e $20 \%$ da população adulta na América do Norte (podendo alcançar taxas como 27\%), o que corresponde a mais de 60 milhões de indivíduos ${ }^{(1-3)}$. Do ponto de vista do impacto populacional, a doença é mais prevalente que uma série de outras afecções crônicas comuns, como a hipertensão, obesidade e diabetes mellitus ${ }^{(4)}$. Ocorre de maneira predominante no sexo feminino $(2: 1)$ e acomete todas as faixas etárias, embora seja mais comum em indivíduos com idade superior a 65 anos ${ }^{(3,4)}$. Considerando-se o impacto médico e socioeconômico, a CI é motivo de mais de 2,5 milhões de consultas médicas anualmente nos EUA, gastos anuais superiores a 2700 dólares por paciente, aumento dos índices de absenteísmo, declínio da produtividade individual e diminuição da qualidade de vida ${ }^{(5-10)}$.

A fisiopatologia da doença é complexa e multifatorial, resultando da combinação direta ou indireta de fatores estruturais e anatômicos, mecânicos, 
metabólicos e funcionais sobre o cólon, reto e ânus. A etiologia é incerta na maioria dos casos, nos quais não são encontradas alterações orgânicas ou anatômicas que justifiquem a apresentação dos sintomas; apenas uma pequena parte dos pacientes apresenta condições subjacentes que justificam o quadro, a exemplo de obstruções mecânicas do trato digestivo, doença de Hirschsprung, afecções neurogênicas, diabetes, lesões à medula espinal, acidentes vasculares encefálicos, entre outras ${ }^{(11)}$.

Embora a definição da doença com base na apresentação dos sintomas muitas vezes seja divergente entre médicos e pacientes ${ }^{(11)}$, há critérios consensuais que auxiliam na padronização do diagnóstico. Os critérios diagnósticos para a CI, recentemente atualizados como Critérios de Roma III ${ }^{(12)}$, estabelecem a necessidade de apresentação, por parte do paciente, de dois ou mais dos seguintes sintomas no período dos últimos 3 meses, com início das manifestações em no mínimo 6 meses antes do diagnóstico, em pelo menos $25 \%$ das defecações: esforço evacuatório, fezes duras ou em cíbalos, sensação de evacuação incompleta, sensação de bloqueio anorretal e necessidade de manobras manuais facilitadoras; outro critério diagnóstico, considerando-se o número de evacuações, corresponde a uma frequiência de evacuações inferior a 3 vezes por semana; além disso, a eliminação de fezes aquosas raramente deverá se apresentar sem o uso de laxantes, e deve haver insuficiência de critérios para o estabelecimento do diagnóstico de Síndrome do Intestino Irritável.

Um fator importante a ser considerado é a associação entre a CI e condições psicológicas particulares, como estresse, ansiedade e depressão ${ }^{(12)}$; tais manifestações parecem predispor à CI, com possível implicação na patogênese da doença ${ }^{(13)}$. De maneira similar, é conhecido que profissionais de saúde, dada a natureza de seu exercício profissional, apresentam maior suscetibilidade ao desenvolvimento de distúrbios de natureza psicológica ${ }^{(14)}$; diversos estudos revelam maior prevalência de estresse e depressão entre médicos e estudantes de medicina ${ }^{(15,16)}$. Dada essa relação, é possível que estudantes de medicina apresentem maior prevalência de CI se comparados à população geral; além disso, há uma série de outros fatores concorrentes que poderiam justificar tal hipótese na população em questão, tais como sedentarismo e hábitos alimentares, fatores tradicionalmente associados à $\mathrm{CI}^{\left({ }^{(8)}\right.}$. Há, ainda, uma escassez de estudos no Brasil no que se refere à prevalência de CI entre adultos jovens.
Dessa forma, os objetivos do presente estudo foram analisar a prevalência e a relevância de variantes de CI entre estudantes de uma faculdade de medicina do noroeste paulista, e comparar os resultados obtidos com dados epidemiológicos disponíveis na literatura médica.

\section{PACIENTES E MÉTODOS}

Realizou-se um estudo transversal através da aplicação de questionários individuais a 360 estudantes de Medicina de uma única instituição de ensino do Noroeste paulista, todos com idade superior a 18 anos, e independentemente do sexo. O questionário continha questões objetivas acerca de constipação intestinal, com o objetivo de se avaliar o preenchimento dos critérios diagnósticos de Roma III para CI; foram avaliadas variáveis como sexo, idade, história de constipação em algum momento da vida (assim como início e duração), frequência de evacuações diárias e semanais, presença e frequência de fezes endurecidas ou em cíbalos, necessidade e frequência de esforço evacuatório, sensação e frequência de evacuação incompleta e obstrução anorretal, necessidade e frequência de manobras digitais facilitadoras da evacuação; avaliou-se ainda história de uso de laxativos, sedentarismo, ingesta de fibras e líquidos e obediência ao reflexo gastrocólico. A avaliação do tipo de fezes eliminadas mais frequentemente pelo indivíduo se deu através da escala de Bristol ${ }^{(17)}$, variando de endurecidas ou em cíbalos (tipo 1) a totalmente aquosas e sem pedaços sólidos (tipo 7).

A realização do estudo foi aprovada por um Comitê de Ética em Pesquisa, e todos os participantes da pesquisa assinaram Termo de Consentimento Livre e Esclarecido.

Para a análise estatística das variáveis consideradas no presente estudo foram utilizadas técnicas de estatística descritiva para o estabelecimento de frequências e das características de base da amostra; os dados encontrados são mostrados em médias e percentuais. As relações entre os dados dos critérios diagnósticos e as características de vida da população estudada foram avaliadas através do teste exato de Fisher e do qui-quadrado. Considerou-se estatisticamente significante um valor $\mathrm{p}<0,05$. Todos os testes estatísticos foram realizados através do programa Systat 12 for Windows (Systat Software (), Inc. 2007). 


\section{RESULTADOS}

Dos 360 indivíduos aos quais se aplicou o questionário, $150(42 \%)$ responderam às questões apresentadas e tiveram seus dados incluídos no estudo. Houve predominância de participantes do sexo feminino (60\%), com média de idade de 23,5 anos, variando entre $18 \mathrm{e}$ 29 anos. A média do índice de massa corpórea (IMC) feminino foi de 21,89 , e a do IMC masculino foi de 24,98 .

Encontrou-se história relatada de sintomas de CI em algum momento da vida em $72 \%$ das mulheres e em $31 \%$ dos homens. A maior parte dos indivíduos do sexo masculino com história de CI relatou ocorrência na infância, não se estendendo ao presente (13\%), ao passo que entre o sexo feminino o período de maior prevalência foi o atual, sem acometimento prévio na infância (38\%). A taxa geral de prevalência de CI definida mediante preenchimento dos critérios diagnósticos de Roma III foi de $35 \%$; considerando-se a distribuição por sexo, a taxa foi de $55 \%$ entre as mulheres e de $18 \%$ entre os homens. A maior prevalência de constipação intestinal entre mulheres foi estatisticamente significante $(\mathrm{p}<0,0001)$.

Quanto ao número de evacuações diárias, a maioria dos participantes relatou frequência de até uma vez, sendo esse valor de $86 \%$ entre o sexo feminino e de 53\% entre o sexo masculino; a menor frequência de evacuações diárias entre pessoas do sexo feminino foi estatisticamente significante $(p=0,0001)$. A frequência predominante de evacuações por semana foi de até quatro vezes em ambos os sexos. As frequências evacuatórias diárias e semanais correlacionadas ao sexo encontras-se nas Tabelas $1 \mathrm{e}$ 2 , respectivamente.
Dos critérios de Roma III avaliados através do questionário, a necessidade de esforço para evacuar é o que apresenta maior prevalência no grupo do sexo masculino (33\%). Já entre o sexo feminino, embora o esforço evacuatório também esteja presente em alta prevalência (48\%), o aspecto endurecido ou em cíbalos das fezes representa o critério mais frequentemente encontrado (52\%). A Tabela 3 aloca percentualmente os indivíduos de acordo a frequência de apresentação dos critérios de Roma III e o sexo.

Tabela 1 - Número de evacuações por dia de acordo com gênero.

\begin{tabular}{lcc}
\hline & $\begin{array}{c}\text { Sexo } \\
\text { Feminino* }\end{array}$ & $\begin{array}{c}\text { Sexo } \\
\text { Masculino } \dagger\end{array}$ \\
\hline Até 1 evacuação & $86 \%$ & $53 \%$ \\
2 evacuações & $10 \%$ & $33 \%$ \\
3 evacuações & $1 \%$ & $3 \%$ \\
4 ou mais evacuações & - & - \\
\hline
\end{tabular}

* Dados incompletos: $3 \%$.

$\dagger$ Dados incompletos: $11 \%$.

Tabela 2 - Número de evacuações por semana de acordo com gênero.

\begin{tabular}{lcc}
\hline & $\begin{array}{c}\text { Sexo } \\
\text { Feminino }\end{array}$ & $\begin{array}{c}\text { Sexo } \\
\text { Masculino }\end{array}$ \\
\hline Até 1 evacuação & - & - \\
2 evacuações & $9 \%$ & - \\
3 evacuações & $19 \%$ & $3 \%$ \\
4 ou mais & $72 \%$ & $97 \%$ \\
\hline
\end{tabular}

Tabela 3 - Critérios de Roma III de acordo com gênero.

\begin{tabular}{lcccc}
\hline & \multicolumn{2}{c}{ Sexo Feminino } & \multicolumn{2}{c}{ Sexo Masculino } \\
\cline { 2 - 5 } & Sim & Não & Sim & Não \\
\hline Fezes endurecidas ou em cíbalos & $52 \%$ & $48 \%$ & $25 \%$ & $75 \%$ \\
Esforço para evacuar & $48 \%$ & $52 \%$ & $33 \%$ & $67 \%$ \\
Sensação de evacuação incompleta * & $38 \%$ & $62 \%$ & $18 \%$ & $80 \%$ \\
Sensação de obstrução anorretal & $29 \%$ & $71 \%$ & $10 \%$ & $90 \%$ \\
Manobras manuais para facilitar evacuação & $8 \%$ & $92 \%$ & $3 \%$ & $97 \%$ \\
\hline
\end{tabular}

*Dados incompletos entre o sexo masculino: $2 \%$. 
Hábitos de vida relacionados a uma maior frequência de CI também foram analisados. Inibir o reflexo gastrocólico é o que aparece em maior prevalência em ambos os sexos (55\% entre o sexo masculino e 54\% entre o feminino). A Tabela 4 dispõe a frequência de hábitos de vida relacionados à $\mathrm{CI}$ de acordo com o gênero do entrevistado, assim como a significância estatística da diferença entre os dois grupos comparados; apenas o maior uso de laxativos entre mulheres se revelou estatisticamente significante $(\mathrm{p}=0,0005)$.

$\mathrm{O}$ aspecto das fezes segundo a escala de Bristol revelou que $6 \%$ das mulheres apresentam o tipo 1 , relacionado ao trânsito intestinal mais lento dentre todos os tipos. O tipo 3 (forma de salsicha com fissuras superficiais) foi o mais frequente (encontrado em $42 \%$ das mulheres e em $50 \%$ dos homens). Considerandose o número total de indivíduos alocados em dois grupos segundo o tipo das fezes (1-3, 4-7), o sexo feminino apresentou maior frequência de fezes dos tipos $1 \mathrm{a}$ 3 em comparação ao sexo masculino; a diferença foi estatisticamente significante $(p=0,01)$. A Tabela 5 mostra a frequência dos tipos de fezes de acordo com o gênero.

\section{DISCUSSÃO}

Embora a CI seja considerada muitas vezes apenas uma diminuição da frequência evacuatória, essa doença se manifesta através de um amplo espectro de sintomas que variam não apenas em complexidade, mas também em cronicidade e intensidade; além disso, é necessário que se considere que as manifestações de tais sintomas estão vinculadas à percepção individual do paciente, algo intensamente variável. Essas observações são necessárias para que se crie uma visão da necessidade de individualização na rotina de investigação diagnóstica e na instituição da terapêutica.

A compreensão dos mecanismos fisiopatológicos subjacentes às variadas formas de CI muitas vezes permite uma caracterização adequada dos sintomas do paciente. Pacientes que apresentam CI de trânsito lento e de trânsito normal (CI funcional), por exemplo, queixam-se mais frequentemente de esforços evacuatórios, redução na frequência das evacuações e endurecimento das fezes, embora os resultados de exames do trânsito intestinal sejam diferentes entre os dois grupos; já os pacientes com alterações defecatórias (obstruções anorretais ou dissinergia da musculatura pélvica e/ou abdominal, por exemplo) relatam esforços evacuatórios mais intensos, sensação de evacuação incompleta e muitas vezes recorrem a manobras digitais ${ }^{(11)}$.

No mesmo sentido, é fundamental em termos clínicos e de pesquisa o estabelecimento de critérios diagnósticos para a doença; os critérios de Roma cum-

Tabela 5 - Número de evacuações por semana de acordo com gênero.

\begin{tabular}{|c|c|c|}
\hline & Sexo Feminino & Sexo Masculino \\
\hline Tipo 1 & $6 \%$ & - \\
\hline Tipo 2 & $24 \%$ & $2 \%$ \\
\hline Tipo 3 & $42 \%$ & $50 \%$ \\
\hline Tipo 4 & $27 \%$ & $44 \%$ \\
\hline Tipo 5 & $1 \%$ & $2 \%$ \\
\hline Tipo 6 & - & $2 \%$ \\
\hline Tipo 7 & - & - \\
\hline
\end{tabular}

Tabela 4 - Frequência de hábitos de vida relacionados à CI de acordo com gênero.

\begin{tabular}{lccccc}
\hline & \multicolumn{2}{c}{ Sexo Feminino } & \multicolumn{2}{c}{ Sexo Masculino } & \multirow{2}{*}{ Valor p } \\
\cline { 2 - 4 } & Sim & Não & Sim & Não & \\
\hline Uso atual ou prévio de laxativos & $46 \%$ & $54 \%$ & $3 \%$ & $97 \%$ & 0,0005 \\
Sedentarismo & $46 \%$ & $54 \%$ & $28 \%$ & $72 \%$ & 0,13 \\
Ingesta reduzida de fibras* & $37 \%$ & $63 \%$ & $48 \%$ & $50 \%$ & 0,17 \\
Ingesta inadequada de líquidos $\dagger$ & $44 \%$ & $56 \%$ & $37 \%$ & $62 \%$ & 0,48 \\
Não obedece ao reflexo gastrocólico & $54 \%$ & $46 \%$ & $55 \%$ & $45 \%$ & 0,94 \\
\hline
\end{tabular}

* Dados incompletos entre o sexo masculino: $2 \%$.

$\dagger$ Dados incompletos entre o sexo masculino: $1 \%$. 
prem adequadamente esse desígnio, ao mesmo tempo em que consideram a larga variação dos sintomas da CI e os alocam de acordo com parâmetros cronológicos.

Os resultados dos estudos epidemiológicos de prevalência da CI dependem consideravelmente da definição da doença adotada na metodologia. Em 2004 um estudo epidemiológico comparou a prevalência de CI segundo diferentes critérios diagnósticos aplicados aos mesmos participantes da pesquisa; as taxas de prevalência se mostraram diferentes, sendo mais altas (próximas a 30\%) quando considerado o auto-relato de CI por parte do paciente, e mais baixas quando considerados os critérios de Roma I $(19,2 \%)$ e II $(14 \%)$ (18). De maneira análoga, em nosso estudo o auto-relato de CI foi consideravelmente maior em relação ao preenchimento dos critérios diagnósticos de Roma III, tanto entre homens $(31 \% \times 18 \%$, respectivamente) quanto entre mulheres $(72 \%$ x $55 \%$, respectivamente).

Considerando-se a comparação dos resultados, é importante ressaltar que algumas limitações e mesmo alguns vieses podem aparecer quando se analisam estudos realizados entre diferentes populações, de diferentes faixas etárias, e que usam diferentes critérios diagnósticos; ainda assim, há certa dificuldade em se encontrar estudos com características homogêneas que facilitem comparações. Uma revisão de literatura recentemente publicada ${ }^{(19)}$ considerando 21 estudos conduzidos entre 34 grupos populacionais diferentes revelou que nenhum dos estudos havia usado os critérios de Roma III na determinação da prevalência de CI; isso talvez se deva ao fato de a classificação ser recente.

De qualquer forma, em termos de CI, a mudança mais importante ocorrida na transição dos critérios de Roma II para III foi quanto à cronologia dos sintomas ${ }^{(20)}$, tornando necessário um tempo maior de apresentação dos sintomas para o estabelecimento do diagnóstico; assim, é de se esperar que estudos que usam os critérios de Roma II teoricamente teriam maior chance de apresentar maior número de casos diagnosticados, já que também incluiriam pacientes com tempo de doença insuficiente para preenchimento dos critérios cronológicos de Roma III. Nesse sentido, é possível afirmar que, de fato, a prevalência de CI encontrada no presente estudo foi maior que a observada entre a população geral na literatura, mesmo segundo os critérios de Roma II.
Outro fato que merece destaque foi a elevada prevalência de CI encontrada entre o sexo feminino no presente estudo. A associação epidemiológica entre sexo feminino e maiores taxas de prevalência de CI é amplamente relatada na literatura ${ }^{(3,18,21,22)}$. Uma das explicações para o fato seriam os fatores hormonais, considerando-se que durante a fase lútea do ciclo menstrual o risco de manifestações de sintomas de CI estaria aumentado em consequência da ação da progesterona ${ }^{(23)}$, a qual também é encontrada em níveis elevados durante os dois últimos trimestres da gestação (fator epidemiologicamente associado à CI) ${ }^{(24,25)}$; fatores como danos à musculatura do assoalho pélvico durante cirurgias ginecológicas e obstétricas também poderiam justificar tal prevalência ${ }^{(26)}$.

Outro fator epidemiológico importante a ser considerado em estudos de prevalência é a faixa etária e a média de idade do grupo estudado; a amostra do presente estudo corresponde a adultos jovens, grupo pouco analisado em termos de prevalência de CI. Um estudo australiano de 2000 comparou taxas de prevalências segundo grupos etários (18 a 23, 45 a 50 e 70 a 75 anos), revelando taxas de prevalência de $14,1 \%, 26,6 \%$ e $27 \%$, respectivamente ${ }^{(26)}$; estima-se que a associação positiva entre CI e idade possa ser decorrente das etiologias secundárias da $\mathrm{CI}{ }^{(27)}$. Esses dados mostram o impacto epidemiológico da CI mesmo em grupos mais jovens, ainda que as taxas relatadas sejam inferiores àquelas observadas em faixas etárias mais elevadas; a prevalência de CI encontrada no presente estudo é elevada mesmo quando confrontada segundo idade.

Por fim, ainda que tenha considerado uma amostra específica de indivíduos, e embora o uso de questionários em estudos epidemiológicos possa se associar a discrepâncias oriundas da percepção do participante a respeito da doença, o presente estudo se utilizou de critérios diagnósticos amplamente fundamentados do ponto de vista clínico e de pesquisa, e contou com uma população que possui algum tipo de conhecimento técnico sobre a doença ou, no mínimo, apresenta fáceis condições de acesso a informações médicas e científicas relacionadas ao tema; assim sendo, tendo sido estabelecidos dados que merecem consideração do ponto de vista epidemiológico, as causas da prevalência aumentada entre essa população deverão ser objeto de estudos mais aprofundados futuramente. 
Rev bras Coloproct Abril/Junho, 2010
Prevalência de Constipação Intestinal entre Estudantes de Medicina de uma Instituição no Noroeste Paulista Carmen Trisóglio e Cols.
Vol. 30

\section{CONCLUSÕES}

1. Observou-se elevada frequência quanto à história de sintomas de CI entre os estudantes de medicina analisados.

2. A prevalência de CI na população estudada é superior à relatada entre a população geral na literatura médica, mesmo ao se considerar sexo e faixa etária.
3. A prevalência de CI preenchendo os critérios de Roma III é maior entre estudantes do sexo feminino, sendo a diferença estatisticamente significante.

4. Estudantes do sexo feminino apresentam menor frequência de evacuações diárias, maior frequência de fezes dos tipos 1 a 3 , segundo a escala de Bristol, e maior frequência de uso de laxativos, sendo as diferenças estatisticamente significantes.

\begin{abstract}
Introduction: Constipation is a highly prevalent disease in the Western world. It has a complex and multifactorial etiology, and courses with important impacts from a medical and socio-economical point of view. It is possible that health professionals and medical students, given the nature of their professional activities, trend to present higher rates of the disease in comparison to the general population. Objectives: To determine the prevalence rates of constipation among students of a single medical school according to diagnostic criteria in the literature, and to compare them with those reported among the general population. Patients and Methods: A transversal study was carried out with a sample of 360 medical students of a single medical school in Northwestern São Paulo State, by means of a self-administered questionnaire; a total of 150 students (42\%) completed the questionnaire; definition of constipation was based on the Rome III diagnostic criteria. Results: The overall prevalence rate of constipation was $35 \%$, with predominance of the disease among female $(55 \%$; $p<0.0001)$; female students also had a lower frequency of daily evacuations and a higher frequency of laxative use $(\mathbf{p}<0.05)$. Conclusions: The prevalence rate of constipation in the studied population was higher than that reported among general population in several studies, and a more accurate search about this fact will be object of further studies.
\end{abstract}

Key words: Constipation; Gastrointestinal Tract; Epidemiology; Prevalence; Students, Medical.

\section{REFERÊNCIAS}

1. Pare P, Ferrazzi S, Thompson WG, Irvine EJ, Rance L. An epidemiological survey of constipation in Canada: definitions, rates, demographics, and predictors of health care seeking. Am J Gastroenterol 2001;96:3130-7.

2. Stewart WF, Liberman JN, Sandler RS, Woods MS, Stemhagen A, Chee E, et al. Epidemiology of constipation (EPOC) study in the United States: relation of clinical subtypes to sociodemographic features. Am J Gastroenterol 1999;94:3530-40.

3. Higgins PD, Johanson JF. Epidemiology of constipation in North America: a systematic review. Am J Gastroenterol 2004;99:750-9.

4. Pleis JR, Lethbridge-Cejku M. Summary health statistics for U.S. adults: National Health Interview Survey, 2005. Vital Health Stat 10 2006;232:1-153.

5. Johanson JF, Sonnenberg A, Koch TR. Clinical epidemiology of chronic constipation. J Clin Gastroenterol 1989;11:525-36.

6. Johanson JF, Kralstein J. Chronic constipation: a survey of the patient perspective. Aliment Pharmacol Ther 2007;25:599608.

7. Martin BC, Barghout V, Cerulli A. Direct medical costs of constipation in the United States. Manag Care Interface 2006;19:43-9.
8. Chang L, Toner BB, Fukudo S, Guthrie E, Locke GR, Norton $\mathrm{NJ}$, et al. Gender, age, society, culture, and the patient's perspective in the functional gastrointestinal disorders. Gastroenterology 2006;130:1435-46.

9. Sonnenberg A, Koch TR: Physician visits in the United States for constipation: 1958 to 1986. Dig Dis Sci 1989;34:60611.

10. Rantis PC Jr, Vernava AM 3rd, Daniel GL, Longo WE: Chronic constipation-is the work-up worth the cost? Dis Colon Rectum 1997;40:280-6.

11. Lembo A, Camilleri M. Chronic Constipation. N Engl J Med 2003;349:1360-8.

12. Haug TT, Mykletun A, Dahl AA. Are anxiety and depression related to gastrointestinal symptoms in the general population? Scand J Gastroenterol 2002;37:294-8.

13. Cheng C, Chan AO, Hui WM, Lam SK. Coping strategies, illness perception, anxiety and depression of patients with idiopathic constipation: a population-based study. Aliment Pharmacol Ther 2003;18:319-26.

14. Nogueira-Martins LA. Saúde mental dos profissionais de saúde. Rev Bras Med Trab 2003;1:56-68.

15. Goebert D, Thompson D, Takeshita J, Beach C, Bryson P, Ephgrave K, et al. Depressive symptoms in medical students and residents: a multischool study. Acad Med 2009;84:23641 . 
16. Dyrbye LN, Thomas MR, Shanafelt TD. Systematic review of depression, anxiety, and other indicators of psychological distress among U.S. and Canadian medical students. Acad Med 2006;81:354-73.

17. Lewis SJ, Heaton KW. Stool form scale as a useful guide to intestinal transit time. Scand J Gastroenterol 1997;32:920-4.

18. Garrigues V, Galvez C, Ortiz V, Ponce M, Nos P, Ponce J. Prevalence of constipation: agreement among several criteria and evaluation of the diagnostic accuracy of qualifying symptoms and self-reported definition in a population-based survey in Spain. Am J Epidemiol 2004;159:520-6.

19. Peppas G, Alexiou VG, Mourtzoukou E, Falagas ME. Epidemiology of constipation in Europe and Oceania: a systematic review. BMC Gastroenterol 2008;8:5.

20. Drossman DA, Dumitrascu DL. Rome III: New standard for functional gastrointestinal disorders. J Gastrointestin Liver Dis 2006;15:237-41.

21. Campbell AJ, Busby WJ, Horwath CC. Factors associated with constipation in a community based sample of people aged 70 years and over. J Epidemiol Community Health 1993;47:23-6.

22. Walter S, Hallböök O, Gotthard R, Bergmark M, Sjödahl R. A population-based study on bowel habits in a Swedish community: prevalence of faecal incontinence and constipation. Scand J Gastroenterol 2002;37:911-6.
23. Turnbull GK, Thompson DG, Day S, Martin J, Walker E, Lennard-Jones JE. Relationships between symptoms, menstrual cycle and orocaecal transit in normal and constipated women. Gut 1989;30:30-4.

24. Derbyshire EJ, Davies J, Detmar P. Changes in bowel function: pregnancy and the puerperium. Dig Dis Sci 2007;52:324-8.

25. Baron TH, Ramirez B, Richter JE. Gastrointestinal motility disorders during pregnancy. Ann Intern Med 1993;118:36675.

26. Chiarelli P, Brown W, McElduff P. Constipation in Australian women: prevalence and associated factors. Int Urogynecol J Pelvic Floor Dysfunct 2000;11:71-8.

27. Brandt LJ, Prather CM, Quigley EM, Schiller LR, Schoenfeld P, Talley NJ. Systematic review on the management of chronic constipation in North America. Am J Gastroenterol 2005; 100(Suppl. 1): S5-S21.

Endereço para correspondência:

JOÃO GOMES NETINHO

Rua San Francisco, 481.

Condomínio Débora Cristina

São José do Rio Preto - SP

CEP: $15090-030$

E-mail :jgnetinho@ riopreto.com.br 